\title{
Comparison of three different surface plank exercises on core muscle activity
}

\author{
Jin Lee ${ }^{a}, K_{\text {wanghyun Jeong }}{ }^{b}$, Hyuna Lee $^{b}$, Jaeyeon Shin ${ }^{b}$, Jaelim Choi ${ }^{b}$, Seungbeom Kang ${ }^{b}$, Byoung-Hee Lee ${ }^{b}$ \\ ${ }^{a}$ Department of Physical Therapy, Hyun-Myoung Medical Center, Seoul, Republic of Korea \\ ${ }^{\mathrm{b}}$ Department of Physical Therapy, College of Health and Welfare, Sahmyook University, Seoul, Republic of Korea
}

Objective: This study compared the muscle activities of the erector spinae (ES), the external oblique (EO), and the rectus abdominis (RA) on three different surfaces. The purpose of this study was to determine which surface induces the highest muscle activity during the plank exercises. The information from this study can be used to recommend plank exercises to athletes and patients with weak core muscles.

Design: Cross-sectional study.

Methods: The subjects include 20 adult males attending S University in Seoul. Participants completed each plank exercise on three different surfaces. To measure muscle activities, researchers used the values from electromyography. The measurement excluded the initial two and final two seconds and collected information on the RA, EO, and ES in each posture of each subject.

Results: The left external oblique showed significant differences between the plank position on stable ground (ST) and the plank position using a suspension device (SL) $(p<0.05)$ and between the plank position on the unstable ground (US) and SL ( $p<0.05$ ). The right rectus abdominis and left rectus abdominis displayed statistically significant differences between the ST and the US $(p<0.05)$ and between the ST and the SL $(p<0.05)$. The right erector spinae had a statistically significant difference between ST and US $(p<0.05)$.

Conclusions: The plank exercise strengthens the core muscles effectively, and muscle activity is related to the posture of the exercise and the location of the muscle. These results suggest that plank exercises improve muscle activities. Additionally, plank exercises can be applied to general medical care.

Key Words: Electromyography, Isometric exercise, Posture

\section{Introduction}

Core muscles are the deep and shallow muscles of the trunk; they stabilize the spinal column, align the body, and enhance performance when the extremities move [1]. Weaknesses in core muscles can cause changes in body configuration and compress the posterior joints of the lumbar spine. Additionally, excessive anterior or posterior leaning of the pelvis accompanied by the tension of the thoracolumbar fascia may result in increased shocks on the flanks and lower extremities [2].

Core exercises can prevent injuries in sports and rehabili- tation treatment by maximizing muscle power and endurance [3]. Exercises to strengthen core muscles include the crunch exercise, the bridge exercise, and the plank exercise [4]. Among them, the plank exercise estimates and strengthens the stability of the core muscles. Core muscle stability is crucial for preventing injuries to the knees, hip joints, and the lumbar spine. Moreover, core muscle stability relieves back pain by maintaining proper alignment for posture and gait [5].

The plank exercise is a posture designed to use body weight to resist gravity; it can be performed on various surfaces, in variable positions, with devices, and impacts multiple

Received: 1 March, 2016 Revised: 14 March, 2016 Accepted: 14 March, 2016

Corresponding author: Byoung-Hee Lee

Department of Physical Therapy, College of Health and Welfare, Sahmyook University, 815 Hwarang-ro, Nowon-gu, Seoul 01795, Republic of Korea Tel: 82-2-3399-1634 Fax: 82-2-3399-1639 E-mail: 3679@syu.ac.kr

(c) This is an Open-Access article distributed under the terms of the Creative Commons Attribution Non-Commercial License (http://creativecommons.org/licens es/by-nc/4.0) which permits unrestricted non-commercial use, distribution, and reproduction in any medium, provided the original work is properly cited.

Copyright $@ 2016$ Korean Academy of Physical Therapy Rehabilitation Science 
joints. Plank exercises on the unstable surface using dynamic cushions for the lower extremities can induce greater activities than plank exercises of the upper extremities on the unstable or on the stable surface [6]. Plank exercises using a suspension device generates greater core muscle activities compared to plank exercises performed on stable or unstable surfaces [5].

Few studies have comprehensively examined core muscle activity in the plank posture on stable and unstable surfaces, or while using a suspension device. This study compared the muscle activities of the erector spinae (ES), external oblique (EO), and rectus abdominis (RA) on three different surfaces while subjects performed plank exercises. The purpose of this study is to determine which surface induces the highest muscle activity during the plank exercises. The information from this study can be used to recommend plank exercises to athletes and patients with weak core muscles.

\section{Methods}

\section{Subjects}

The subjects include 20 adult males attending Sahmyook University in Seoul. The present study was approved by Sahmyook University Institutional Review Board and each subject was able to follow instructions and gave informed consent by signing an approved consent form; thus, the rights of human subjects were protected. The selection criteria included individuals who: understood the study, a body mass index between 18.5 and $25 \mathrm{~kg} / \mathrm{m}^{2}$, volunteered to join, and had exercised less than three times a week. The exclusion criteria included individuals who had: exercise limitations from a physician, used steroid or protein supplements, drank more than two bottles of alcohol a week, or could not maintain the plank posture.

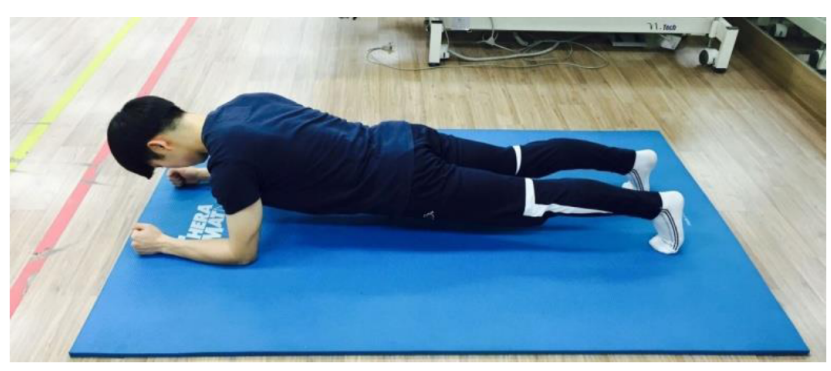

Figure 1. The plank position on stable ground.

\section{Procedures}

Twenty subjects practiced the plank position and warmed up before the researchers measured core muscle activity. After the warm-up, five subjects were withdrawn from the study due to immature posturing. The order of the three exercises was randomly assigned to the fifteen subjects.

The plank position utilized had the same initial posture. Both forearms maintained contact with the ground while the hands made fists and the elbows maintained a distance of 30 $\mathrm{cm}$ apart. The researchers instructed the subjects to protract the scapulas and maintain 90-degree angles at the ankles. The abdomen was contracted using the abdominal drawing-in maneuver method; the heights of the shoulders and hips from the ground were keep at $25 \mathrm{~cm}$. The plank exercise on the stable ground required that the feet touch the ground (Figure 1) [7]. The plank exercise on the unstable ground utilized a dynamic cushion at the bottom of each foot; the air pressure was the same in the two cushions. The subjects were required to maintain the posture [8] for 15 seconds (Figure 2). In the plank exercise using a suspension device, each ankle was tied to the straps of the device. The heights of the ankles from the ground were maintained at $40-50 \mathrm{~cm}$. Support was provided as needed so that the subject did not excessively tilt towards the front or swing from side to side (Figure 3) [5]. All subjects were advised to breathe regularly

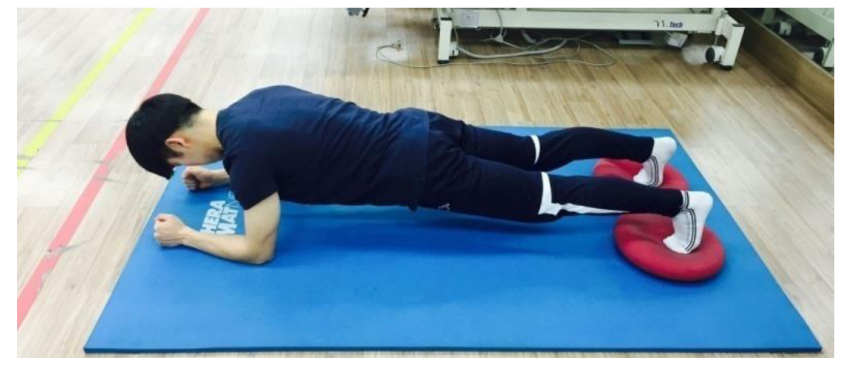

Figure 2. The plank position on unstable ground.

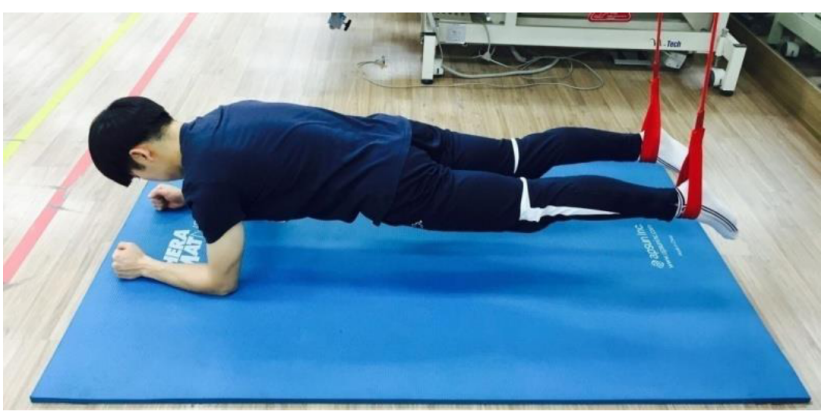

Figure 3. The plank position using a suspension device. 
while performing plank exercises.

Each experiment consisted of a three minute warm up and three sets of a plank exercise maintained for fifteen seconds. A break was given for 30 seconds between each set; a threeminute break was given between performing each different plank exercise. To measure muscle activities, researchers used the values from electromyography (TELEMYO 2400T $\mathrm{G} 2^{\circledR}$; NORAXON, Scottsdale, AZ, USA). The measurement excluded the initial two and final two seconds and collected information on the RA, EO, and ES in each posture of each subject. The greatest value of electromyography when each muscle was contracted to its maximum was regarded as the maximal voluntary isometric contraction (MVIC). The MVIC was measured three times for five seconds each by having the participant resist the researcher's manual resistance. To help assess manual resistance to the RA, the subject lifted their upper body while flexing the hip joints and the knees; the evaluator applied resistance on both shoulders. For the EO, the subject rotated the erected upper body to the left and right; the evaluator applied resistance on both shoulders. For the ES, the subject laid down on their abdomen, placed their hands with locked fingers on the back of the head, and lifted the upper body up as much as possible while an evaluator helped to fix the lower body and another evaluator applied resistance on both shoulders [5].

The electromyography leads were placed on $2 \mathrm{~cm}$ lateral points from the umbilicus with $3 \mathrm{~cm}$ longitudinal intervals for the RA. For the EO, the leads were placed between the 12th rib and the iliac crest, along the fibers of the EO just above the anterior superior iliac spine with $2 \mathrm{~cm}$ intervals. For the ES, the leads were placed on $2 \mathrm{~cm}$ lateral points from the spinous process of the level of the iliac crest with $2 \mathrm{~cm}$ longitudinal intervals [9].

\section{Analysis}

The average and maximum values were calculated using PASW ver. 18.0 (IBM Co., Armonk, NY, USA). Researchers used Mauchly's Test of Sphericityto determine if the variances of the differences between all possible pairs of groups were equal. Researchers analyzed the data using repeated measures analysis of variance to evaluate the differences between the groups. The threshold of the statistical significance was 0.05 .

\section{Results}

The general characteristics of the subjects revealed no significant difference between the samples. The average weight and height of the subjects were $67 \mathrm{~kg}$ and $174.5 \mathrm{~cm}$, respectively.

Performing a plank exercise on three different surfaces revealed statistically significant differences in the muscle activities of the left external oblique (LEO), right rectus abdominis (RRA), left rectus abdominis (LRA), and right erector spinae (RES) $(p<0.05)$; there were no significant muscle activity differences in the right external oblique (REO) and left erector spinae (LES) (Table 1, Figure 4). The LEO showed significant differences between the plank position on stable ground (ST) and the plank position using a suspension device (SL) $(p=0.028)$ and between the plank position on the unstable ground (US) and SL ( $p=0.047)$; however, there were no significant differences between ST and US. The RRA displayed statistically significant differences between the ST and the US $(p=0.012)$ and between the ST and the SL ( $p=0.034$ ); however, there were no significant differences between the US and the SL. The LRA exhibited statistically significant differences between ST and US

Table 1. Comparison of muscle activities between groups

$(\mathrm{N}=15)$

\begin{tabular}{cccccc}
\hline Variable & ST-plank & US-plank & SL-plank & $F$ & $p$ \\
\hline REO (\%MVIC) & $29.61(16.19)$ & $33.60(23.07)$ & $38.93(28.86)$ & 2.210 & 0.149 \\
LEO (\%MVIC) & $33.58(23.85)$ & $36.32(28.65)$ & $41.03(29.71)$ & 3.627 & 0.040 \\
RRA (\%MVIC) & $20.28(10.83)$ & $24.82(13.26)$ & $27.04(16.78)$ & 3.844 & 0.049 \\
LRA (\%MVIC) & $19.34(11.44)$ & $23.72(14.28)$ & $24.60(14.99)$ & 5.488 & 0.019 \\
RES (\%MVIC) & $5.15(1.43)$ & $5.88(1.86)$ & $6.91(3.49)$ & $8.424^{*}$ & 0.005 \\
LES (\%MVIC) & $5.34(3.03)$ & $6.08(2.74)$ & $5.83(2.35)$ & 3.783 & 0.051 \\
\hline
\end{tabular}

Values are presented as $\mathrm{n}(\%)$ or mean (SD).

ST-plank: the plank position on stable ground, US-plank: the plank position on unstable ground, SL-plank: the plank position using a suspension device, REO: right external oblique muscle, LEO: left external oblique muscle, RRA: right rectus abdominal muscle, LRA: left rectus abdominal muscle, RES: right erector spinal muscle, LES: left erector spinal muscle, MVIC: maximum voluntary isometric contraction. 


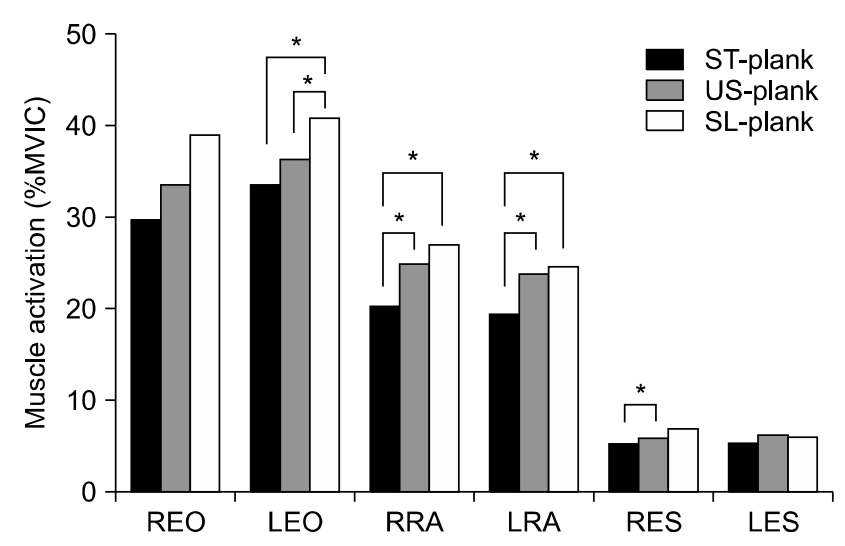

Figure 4. Comparison of muscle activities between groups. ST-plank: the plank position on stable ground, US-plank: the plank position on unstable ground, SL-plank: the plank position using a suspension device, REO: right external oblique muscle, LEO: left external oblique muscle, RRA: right rectus abdominal muscle, LRA: left rectus abdominal muscle, RES: right erector spinal muscle, LES: left erector spinal muscle, MVIC: maximum voluntary isometric contraction $\left({ }^{*} p<0.05\right)$.

( $p=0.004)$ and between ST and SL ( $p=0.028)$; however, there were no significant differences between US and SL. The RES had a statistically significant difference between ST and US $(p=0.005)$.

\section{Discussion}

The purpose of this study was to compare core muscle activities in plank exercises performed on three different surfaces among adult men. Exercises on an unstable surface intensify the activities of the muscles and the cooperation pattern among the stabilizing muscles [10]. Additionally, the exercises provide changed sensory inputs to the muscles and activate the proprioceptors and neuroadaptive mechanisms.

In this study, US and SL showed significant muscle activities in the RA, EO, and ES compared to the ST $(p<0.05)$. Tong et al. [7] measured muscle activities of the RA, EO, and ES in 36 healthy adults with a program consisting of the primary plank position (60 seconds), plank position with one upper extremity lifted (15 seconds each side), plank position with one lower extremity lifted (15 seconds each side), plank position with contralateral extremities lifted together (15 seconds each side), and back to the primary plank position (30 seconds) without rest between cycles. Tong et al. [7] reported significant differences between the plank exercise requiring one upper extremity lifted and the basic plank position $(p<0.05)$, between the plank exercise with one lower extremity lifted and the plank exercise with one upper ex- tremity lifted $(p<0.05)$, and between the plank exercise with contralateral extremities lifted together and the plank exercise with one lower extremity lifted $(p<0.05)$. Czaprowski et al. [11] measured muscle activities in the right RA, EO, and internal oblique (IO) of 33 healthy adults in plank exercises supporting the forearms on the stable ground, using a BOSU ball, and gym balls. The order of the exercises was designated by a random ballot; a one minute break was given between each exercise. As a result, among all of the muscles measured (RA, EO, and IO), significant changes were observed between the plank exercise on the BOSU ball and the plank exercise on stable ground, between the plank exercise on the gym ball and the plank exercise on stable ground, and between the plank exercise on the gym ball and the plank exercise on the BOSU ball $(p<0.05)$. The current study demonstrates a consistent result with Tong et al. [7] and Czaprowski et al. [11] The consistency between all three studies may be because the plank exercise on the unstable surface requires more muscle demands to maintain the elevated position compared to stable surface [5].

In this study, LES showed no significant differences in muscle activity while RES showed significant differences in muscle activity between ST and US and between ST and SL. Tong et al. [7] reported significant differences in the RA and the EO among plank positions with different bases of support. However, the muscle activity of the ES was weak and not included in the timeline measuring the values (10 seconds). Even though the ES is used before and after the plank exercises, it demonstrates very low muscle activity during the plank exercise. The role of the ES in the plank exercise is limited as the plank exercise resists body weight and gravity with the anterior surface of the trunk. Despite the weak muscle activity of the ES, the plank exercise can be used to evaluate and strengthen the core muscles, mainly focusing on the transversus abdominis (TrA). Mok et al. [5] measured and compared the muscle activities of the RA, EO, IO, TrA, and lumbar multifidus (LMF) in 18 healthy adults in the hip addition plank (HAP) using a suspension device, chest press (CP), $45^{\circ}$ row (ROW), and hamstring curl (HC). The EO, IO, and TrA showed the most significant muscle activity in the HAP compared to the $\mathrm{CP}$, ROW, or $\mathrm{HC}$ $(p<0.05)$; interestingly, the $\mathrm{HC}$ induced the greatest muscle activity of the LMF than any other exercise $(p<0.05)$. Czapowski et al. [11] measured and compared the muscle activities of the RA, EO, IO, and TrA in the supine bridge, side bridge, and plank exercise on different surfaces. The greatest muscle activity occurred in the plank exercise 
$(p<0.05)$. Based on the previous studies, the plank exercise strengthens the core muscles effectively, and muscle activity is related to the posture of the exercise and the location of the muscle.

\section{Conflict of Interest}

The authors declared no potential conflicts of interest with respect to the authorship and/or publication of this article.

\section{References}

1. Key J. 'The core': understanding it, and retraining its dysfunction. J Bodyw Mov Ther 2013;17:541-59.

2. Kline JB, Krauss JR, Maher SF, Qu X. Core strength training using a combination of home exercises and a dynamic sling system for the management of low back pain in pre-professional ballet dancers: a case series. J Dance Med Sci 2013;17:24-33.

3. Wilk BR, Stenback JT, Gonzalez C, Jagessar C, Nau S, Muniz A. Core muscle activation during Swiss ball and traditional abdominal exercises. J Orthop Sports Phys Ther 2010;40:538-9; author reply 539-41.

4. Ekstrom RA, Donatelli RA, Carp KC. Electromyographic analy- sis of core trunk, hip, and thigh muscles during 9 rehabilitation exercises. J Orthop Sports Phys Ther 2007;37:754-62.

5. Mok NW, Yeung EW, Cho JC, Hui SC, Liu KC, Pang CH. Core muscle activity during suspension exercises. J Sci Med Sport 2015;18:189-94

6. Do YC, Yoo WG. Comparison of the thicknesses of the transversus abdominis and internal abdominal obliques during plank exercises on different support surfaces. J Phys Ther Sci 2015;27: 169-70.

7. Tong TK, Wu S, Nie J. Sport-specific endurance plank test for evaluation of global core muscle function. Phys Ther Sport 2014; 15:58-63.

8. Schoffstall JE, Titcomb DA, Kilbourne BF. Electromyographic response of the abdominal musculature to varying abdominal exercises. J Strength Cond Res 2010;24:3422-6.

9. Youdas JW, Boor MM, Darfler AL, Koenig MK, Mills KM, Hollman JH. Surface electromyographic analysis of core trunk and hip muscles during selected rehabilitation exercises in the side-bridge to neutral spine position. Sports Health 2014;6:41621.

10. Vera-Garcia FJ, Grenier SG, McGill SM. Abdominal muscle response during curl-ups on both stable and labile surfaces. Phys Ther 2000;80:564-9.

11. Czaprowski D, Afeltowicz A, Gębicka A, Pawłowska P, Kędra A, Barrios C, et al. Abdominal muscle EMG-activity during bridge exercises on stable and unstable surfaces. Phys Ther Sport 2014;15:162-8 